Assiut Scientific Nursing Journal

http://asnj.journals.ekb.eg

http://www.arabimpactfactor.com

\title{
Effect of Educational intervention about Nutrition for Infertile Women on their Knowledge in Preconception Period
}

\author{
Basma G. Mohammed ${ }^{1}$, Walaa H. Ibrahim ${ }^{2}$, Esraa Y. Badran ${ }^{3} \&$ Nour El Hoda M. Mohammed ${ }^{4}$. \\ 1. Clinical Instructor, Obstetric \& Gynecological Nursing, Faculty of Nursing - Sohag University, Egypt. \\ 2. Lecturer of Obstetric \& Gynecological Nursing, Faculty of Nursing, Assiut University, Egypt. \\ 3. Lecturer of Obstetric \& Gynecological Medicine, Faculty of Medicin, Assiut University, Egypt. \\ 4. Assistant Professor of Obstetrics \& Gynecological Nursing, Faculty of Nursing, Sohag University. Egypt.
}

\begin{abstract}
Background: Human fertility affected by many factors as nutrition (unbalanced nutrition and unhealthy diet) be together to affect reproduction security in women. Evidence suggests that nutrition can play an important role in altering fertility-related outcomes in both men and women. Aim: this study aims to assess level of knowledge about nutrition that increases fertility in infertile women, and evaluate effect of educational intervention about nutrition on infertile women's knowledge in preconception period. Methods: Quasi experimental (pre - posttest) research design was carried out in this study. It was conducted at infertility outpatient clinic women's health Hospitals, Assiut University, Egypt. Sample included 100 infertile women. Structured interview questionnaire was used and included two parts: Sociodemographic data and questions to assess knowledge regarding fertility's nutrition. Results: There are statistical significant differences between pretest and posttest regarding total knowledge of infertile women about nutrition, P-Value is 0.001. Conclusion: Educational program about nutrition that may increase fertility is very important to enhance infertile women's knowledge regarding nutrition that may increase and decrease fertility. Recommendations: implementing a continuing educational program for women including counseling skills about importance of fertility nutrition during preconception period.
\end{abstract}

\section{Keywords: Educational Program, Nutrition, Knowledge, Infertile Women \& Preconception Period.}

\section{Introduction}

Infertility is inability to get pregnant after one year of trying (or six months for a woman has 35 years or older). Women who can have pregnant but are not able to get pregnant may also be infertile (U.S. Department of Health \& Human Services, 2019). Infertility influences $10 \%-15 \%$ of couples. This marks it as one of the most common diseases for people aged between 20 and 45 (American Society for Reproductive Medicine, 2019). Infertility considers one of the greatest common challenges confronted by women of reproductive age these days (Rizk \& Olsen, 2016). Human fertility affected by many factors as nutrition and unhealthy diet together can affect reproduction security in women (Erica et al, 2019). Other factors as sexual behavior, consanguinity, culture, endocrinology, instinct, timing, money matters, lifestyle, and feelings (Yoo \& Sobotka, 2018).

According to the Center of Disease Control and Prevention (CDC) the causes of female infertility can be divided into three broad categories including defective ovulation, transport and implantation (CDC, 2013). Evidence suggests that nutrition can play an important role in modifying fertility-related outcomes in equally men and women (Gaskins \& Chavarro, 2018).
There are some key nutrients that directly affect fertility in women includes folic acid, iron, and vitamin E (Philip \& David, 2018). Folic acid it's B vitamin aids to decrease fetus risk of neural tube birth defects such as spina bifida. Every woman needs 400 micrograms daily to cover her requirement. It may be obtained naturally through leafy, dark green vegetables (i.e. spinach), citrus fruits, nuts, legumes, whole grains, fortified bread and cereals (American Pregnancy Association, 2018).

Diet rich in iron decrease risk of ovulatory infertility. Foods greatly rich in iron include naturally dried fruit, chickpeas, beans, lentils, quinoa, kale, broccoli, molasses, and organic red meats. Daily requirement is 27 mg (Hillstrom K., 2018).

Vitamin $\mathrm{E}$ is also a fertility nutrient for women, women require 10 IU daily, as it enhances hormone role in women (American Pregnancy Association, 2019). It can be obtained naturally through oily fish, green leafy vegetables, broccoli, and nuts (U.S. Department of Agriculture, 2019).

It can be obtained naturally through oily fish, green leafy vegetables, broccoli, and nuts (U.S. Department of Agriculture, 2019).

There are nutrients have negative effect on fertility that women should be avoided as caffeine, and trans fats (Audrey \& Jorge, 2018). Caffeine is not good 
for baby, but research is also showing that it can lower fertility by approximately $27 \%$. Caffeine also has a negative effect on overall nutrition because it restricts the body's ability to absorb iron and calcium (Abdallah \& Osama, 2018).

Artificial fats, such as trans fats, can disrupt fertility by increasing insulin insensitivity and inflammation in the body, interfering with ovulation, conception, and early embryonic development (Mary., 2013).

Nurse's play a vital role regarding fertility that involved clinical nursing practices, health education, follow-up treatment, and patient education. This has enhanced the accessibility of health-care facilities, decreased women's exposure to risk, better costeffectiveness and improved customers' capabilities of health-care services (Virpi et al, 2013).

\section{Significance of the study}

Infertility affects a great percentage of the young population worldwide, it is valued to affect $10-15 \%$ of couples after consistent marital life (Khosrorad et al., 2015).

Above the past little decades, many lifestyle elements have been progressively shown to influence women fertility, and they considerably influence the chance of pregnancy and delivery (Homan \& Norman, 2018) Harvard performed a recent study that revealed an $80 \%$ reduction in infertility rates with daily life changes made by changing to a fertility diet. Women, who followed a mixture of five or more lifestyle factors, including exchanging specific aspects of their diets, experienced over 80 percent fewer relative risk of infertility as a result of ovulatory disorders (Hethir, 2019).

Aim of the study

This study aims to

- Assess level of knowledge of infertile women about nutrition that increases fertility.

- Evaluate effect of educational intervention about fertility nutritional needs for infertile women's knowledge.

\section{Research Hypothesis}

The research hypotheses for the study were as follow

Hypothesis 0 (H0): No change in women knowledge regarding nutritional needs and no effect on their diets and pregnancy out comes.

Hypothesis 1 (H1): Increased knowledge about fertility nutritional needs for women, their diets will be rich in fertility foods and improvement in pregnancy out comes.

Subjects \& methods

\section{Research design}

Quasi experimental (pre - posttest) research design was carried out in this study.
Study setting

The study was conducted at infertility outpatient clinic at fifth floor at Assiut Women's Health Hospitals, Assiut University. This setting is one of the most important settings serves all cases from urban and rural areas at Assiut City. Which is a university affiliated hospital providing free health care to outpatient gynecologic clients as well as obstetrics clients. Total annual women who had primary infertility visit outpatient infertility clinics are 400 (official record, 2018). Care is provided by obstetrician, as well as professional and diploma nurses who are responsible for giving nursing care.

\section{Sample}

A purposive sample was included in the study. The sample of this study was 100 infertile women. The sample size was determined according to the following equation

Tools $\mathrm{N}=\quad \frac{\mathrm{N} Z^{2} \sigma^{2}}{\mathrm{Z}^{2} \mathrm{G}^{2}+\mathrm{N}}$

The tools included:

A: Structured Interview Questionnaire: that included two parts:

First part

Personal data

Personal data, medical history for any disease, and current history of infertility and Gynecological data.

Second part

(Assessment knowledge regarding fertility nutrition) which includes

Data related to nutrition that may increase fertility as (folic acid, iron, and vitamin E) and data related to food that forbidden as (caffeine, and trans fats). This part included asking about importance of, its sources, and daily requirements of food that increase fertility as folic acid and iron. And sources of food that had a negative effect on fertility like caffeine.

Knowledge scoring system

Each question was scored as (1) for a correct answer and (zero) for an incorrect answer and don't know. While the total knowledge score was calculated as the following: knowledge was considered poor if the percent score was $<50 \%$ and was average if percent was ( $>50 \%$ to $<75 \%)$ and considered good if percent was $75 \%$ and more.

\section{Content validity and reliability}

The tool was established by 5 expertise (three from the internal medicine \& two staff from the obstetric and gynecological nursing, to review relevance of the tools for, comprehensiveness, understanding and applicability, and they valid it. Whilst the reliability was assessed by measuring its internal consistency using, Alpha Cronbach's test for the tool, questionnaire reliability is 0.84 . 


\section{Administration design}

Approval was obtained from the head of department of IVF unit, at Assiut Women's Health Hospitals, Assiut University. The researcher explained the aim of the study and requesting permission to use the premise for data collection. Also oral consent was obtained from the study participants after explaining the purpose and nature of the study.

Operational design

This included the pilot study and data collection phase.

\section{Pilot study}

A pilot study was carried out in April 2019 to test the feasibility and practicability of the study tools on a group of 10 women. No change was done in the assessment sheet, so the ten sample use of women for the pilot study were included in the main study. According to the results of the pilot study, no modification in questionnaire sheet was done.

\section{Frame work}

Data were collected at the infertility outpatient clinic at Assiut Women's Health Hospitals, Assiut University during the period from May 2019 to October 2019. The tools were filled through interviewing, follow up was ended by November 2019. The study was carried out during morning for all patients. The lecture gave to them in the waiting room and in the secretory room.

\section{Intervention phase}

Pretest was done first to the women to assess their knowledge about nutritional needs in the pre conception period, and then the researcher gave the participants health education about nutritional needs in the form of lecture given to them in groups. Number of women in the group differs according to availability of them in this day. The number of women was differed from 4-7 women in each group. The implemented nutrition educational program was conducted through one theoretical session. The duration of session was around 30 minutes. The lecture gave to them in the waiting room and in the secretory room at infertility outpatient clinic at fifth floor at Assiut Women's Health Hospitals, Assiut University.

After that the women took the content of lecture in a form of pourchore. The content of lecture included fertility food types, importance of each type, sources of food for each one, daily requirements and food forbidden from them in preconception period. The pourchore contents were obtained from national and international reviews, articles, and researches and were translated by the researcher into simple clear understandable arabic language and were rich with pictures about the healthy food. Women were asked to come after 4 weeks to perform posttest.
Post intervention phase (follow up)

After 4 weeks of intervention phase, the researcher again met the women to evaluate the effect of educational intervention on their knowledge through posttest.

\section{Ethical consideration}

The study proposal took agreement from the ethical committee of the faculty of nursing, Assiut University. An official permission to carry out the study was obtained from the responsible authorities from Women Health Hospital after inform them about the aim of the study and to gain the needed support and cooperation.

Informed consent was obtained from the study participants after explaining the purpose and nature of the study. Also, assure them that their participation would not be used against them in any way and they have the right to refuse or to decide to terminate their participation at any time.

\section{Statistical analysis}

Data entry and data analysis were done using SPSS version 18 (Statistical Package for Social Science). Data were presented using descriptive statistics in the form of frequencies and percentages. Also, Mean and standard deviations were calculated. Correlation between variables (Pearson correlation) and statistical significance was considered at $\mathrm{P}$-value $\leq 0.05$. 


\section{Result}

Table (1): Distribution of the studied women according to their sociodemographic data.

\begin{tabular}{|c|c|c|}
\hline Sociodemographic characteristics & No (100) & $\%$ \\
\hline $\begin{array}{l}\text { 1) Age/years } \\
\text { - } 18-24 \text { years } \\
\text { - } 25-34 \text { years } \\
\text { - } 35 \text { to } 45 \text { years }\end{array}$ & $\begin{array}{l}15 \\
64 \\
21\end{array}$ & $\begin{array}{l}15.0 \\
64.0 \\
21.0\end{array}$ \\
\hline $\begin{array}{l}\text { 2) Residence } \\
\text { - Urban } \\
\text { - Rural }\end{array}$ & $\begin{array}{l}57 \\
43 \\
\end{array}$ & $\begin{array}{l}57.0 \\
43.0\end{array}$ \\
\hline $\begin{array}{l}\text { 3) Educational level } \\
\text { - Illiterate } \\
\text { - Read and write } \\
\text { - Basic education } \\
\text { - Secondary } \\
\text { - University }\end{array}$ & $\begin{array}{c}4 \\
12 \\
9 \\
43 \\
32\end{array}$ & $\begin{array}{c}4.0 \\
12.0 \\
9.0 \\
43.0 \\
32.0\end{array}$ \\
\hline $\begin{array}{l}\text { 4) Occupation } \\
\text { - Housewife } \\
\text { - Employed }\end{array}$ & $\begin{array}{l}65 \\
35\end{array}$ & $\begin{array}{l}65.0 \\
35.0\end{array}$ \\
\hline $\begin{array}{l}\text { 5) Type of family } \\
\text { - Nuclear } \\
\text { - Extended }\end{array}$ & $\begin{array}{l}39 \\
61\end{array}$ & $\begin{array}{l}39.0 \\
61.0\end{array}$ \\
\hline $\begin{array}{l}\text { 6) Age at marriage: } \\
\text { - }<20 \text { yrs. } \\
\text { - } 20-25 \text { yrs. } \\
\text { - }>25 \text { yrs. }\end{array}$ & $\begin{array}{l}29 \\
53 \\
18\end{array}$ & $\begin{array}{l}29.0 \\
53.0 \\
18.0\end{array}$ \\
\hline $\begin{array}{l}\text { 7) Duration of marriage: } \\
\bullet<2 \text { yrs. } \\
\bullet 2-5 \text { yrs. } \\
\bullet>5 \text { yrs. }\end{array}$ & $\begin{array}{c}6 \\
39 \\
55\end{array}$ & $\begin{array}{c}6.0 \\
39.0 \\
55.0\end{array}$ \\
\hline Total & 100 & $100.0 \%$ \\
\hline
\end{tabular}

Table (2): Distribution of the studied women according to current infertility \& gynecological data.

\begin{tabular}{|l|c|c|}
\hline \multicolumn{1}{|c|}{ Current infertility \&gynecological data } & No (100) & \% \\
\hline 1) Duration of infertility: & 46 & \\
- 1-5 yrs. & 54 & 56.0 \\
- > 5 yrs. & & \\
\hline 2) Cause of infertility: & 24 & 24.0 \\
- Male. & 32 & 32.0 \\
- Female. & 10 & 10.0 \\
- Mix. & 34 & 34.0 \\
- Unexplained. & & \\
\hline 3) Current gynecological problem & 60 & 60.0 \\
- No & 24 & 24.0 \\
- Polycystic ovary syndrome & 7 & 7.0 \\
- Endometriosis & 1 & 1.0 \\
- Congenital anomaly & 3 & 3.0 \\
- Fibroid & 4 & 4.0 \\
- Defective ovum transport & 1 & 1.0 \\
- Defective sperm transport & $\mathbf{1 0 0}$ & $\mathbf{1 0 0 . 0 \%}$ \\
\hline Total &
\end{tabular}


Table (3): Foods contain folic acid in pre and post test.

\begin{tabular}{|c|c|c|c|c|c|}
\hline \multirow{2}{*}{ Data related to Foods contain folic acid } & \multicolumn{2}{|c|}{ Pre test } & \multicolumn{2}{|c|}{ Post test } & \multirow{2}{*}{ p-value } \\
\hline & No (100) & $\%$ & No (100) & $\%$ & \\
\hline $\begin{array}{l}\text { 1) Foods contain folic acid are important to increase fertility } \\
\text { - Yes } \\
\text { - No } \\
\text { - Don't know }\end{array}$ & $\begin{array}{c}55 \\
7 \\
38\end{array}$ & $\begin{array}{c}55.0 \\
7.0 \\
38.0\end{array}$ & $\begin{array}{l}90 \\
4 \\
6\end{array}$ & $\begin{array}{l}90.0 \\
4.0 \\
6.0\end{array}$ & $0.005 * *$ \\
\hline $\begin{array}{l}\text { 2) Main importance of folic acid for fetus: } \\
\text { - Decrease risk of neural tube defect } \\
\text { - Helps ensure women have a healthy baby } \\
\text { - Don't know/ can't remember. }\end{array}$ & $\begin{array}{l}43 \\
14 \\
43\end{array}$ & $\begin{array}{l}43.0 \\
14.0 \\
43.0\end{array}$ & $\begin{array}{c}85 \\
8 \\
7\end{array}$ & $\begin{array}{c}85.0 \\
8.0 \\
7.0\end{array}$ & $0.02 *$ \\
\hline $\begin{array}{l}\text { 3)Women need to start folic acid intake: } \\
\text { - Before pregnancy. } \\
\text { - During pregnancy } \\
\text { - At no stage (they don't need to). } \\
\text { - Don't know. }\end{array}$ & $\begin{array}{c}50 \\
8 \\
4 \\
38\end{array}$ & $\begin{array}{c}50.0 \\
8.0 \\
4.0 \\
38.0\end{array}$ & $\begin{array}{l}92 \\
6 \\
0 \\
2\end{array}$ & $\begin{array}{l}92.0 \\
6.0 \\
0.0 \\
2.0\end{array}$ & $0.001 * *$ \\
\hline $\begin{array}{l}\text { 4)Food are naturally good sources of folic acid } \\
\text { - Know } \\
\text { - Don't know }\end{array}$ & $\begin{array}{l}42 \\
58 \\
\end{array}$ & $\begin{array}{l}42.0 \\
58.0 \\
\end{array}$ & $\begin{array}{c}100 \\
0 \\
\end{array}$ & $\begin{array}{c}100.0 \\
0.0\end{array}$ & NA \\
\hline $\begin{array}{l}\text { 5) Adequate dose of folic acid needed before or } \\
\text { through pregnancy is: } \\
\text { - } 400 \text { micrograms }(\mathrm{mcg}) \\
\text { - } 200 \text { micrograms }(\mathrm{mcg}) \\
\text { - } 400 \text { milligrams }(\mathrm{mg}) \\
\text { - } 0.4 \text { micrograms }(\mathrm{mcg}) \\
\text { - Don't know. }\end{array}$ & $\begin{array}{c}10 \\
0 \\
1 \\
1 \\
88\end{array}$ & $\begin{array}{c}10.0 \\
0.0 \\
1.0 \\
1.0 \\
88.0\end{array}$ & $\begin{array}{c}65 \\
2 \\
15 \\
0 \\
19\end{array}$ & $\begin{array}{c}64.0 \\
2.0 \\
15.0 \\
0.0 \\
19.0\end{array}$ & $0.005 * *$ \\
\hline Total & 100 & $100.0 \%$ & 100 & $100.0 \%$ & \\
\hline
\end{tabular}

Mc Nemar test used for pair qualitative variables

(NA) no applicable because response rate $100.0 \%$

Table (4): Foods contain iron in pre and post test.

\begin{tabular}{|c|c|c|c|c|c|}
\hline \multirow{2}{*}{ Data related to Foods contain iron } & \multicolumn{2}{|c|}{ Pre test } & \multicolumn{2}{|c|}{ Post test } & \multirow{2}{*}{ p-value } \\
\hline & No (100) & $\%$ & No (100) & $\%$ & \\
\hline $\begin{array}{l}\text { 1) Foods contain iron are important to increase fertility } \\
\text { - Yes } \\
\text { - No } \\
\text { - Don't know }\end{array}$ & $\begin{array}{l}16 \\
4 \\
80\end{array}$ & $\begin{array}{l}16.0 \\
4.0 \\
80.0\end{array}$ & $\begin{array}{c}80 \\
0 \\
20\end{array}$ & $\begin{array}{c}80.0 \\
0.0 \\
20.0\end{array}$ & $0.004 * *$ \\
\hline $\begin{array}{l}\text { 2) Main important to take iron before pregnancy is: } \\
\text { - Helps lower risk of ovulatory infertility } \\
\text { - Helps decreased fertility } \\
\text { - Don’t know/ can’t remember. }\end{array}$ & $\begin{array}{l}10 \\
2 \\
88\end{array}$ & $\begin{array}{c}10.0 \\
2.0 \\
88.0\end{array}$ & $\begin{array}{c}87 \\
0 \\
13\end{array}$ & $\begin{array}{c}87.0 \\
0.0 \\
13.0\end{array}$ & $0.003 * *$ \\
\hline $\begin{array}{l}\text { 3) Food are naturally good sources of iron } \\
\text { - Know } \\
\text { - Don't know }\end{array}$ & $\begin{array}{l}75 \\
25\end{array}$ & $\begin{array}{l}75.0 \\
25.0\end{array}$ & $\begin{array}{c}100 \\
0\end{array}$ & $\begin{array}{c}100.0 \\
0.0\end{array}$ & NA \\
\hline $\begin{array}{l}\text { 4) Adequate dose of iron needed before pregnancy is: } \\
\text { - } 27 \text { milligrams }(\mathrm{mg}) \text {. } \\
\text { - } 27 \text { micrograms (mcg) } \\
\text { - } 0.27 \text { micrograms (mcg). } \\
\text { - Don't know. }\end{array}$ & $\begin{array}{l}15 \\
10 \\
1 \\
74\end{array}$ & $\begin{array}{c}15.0 \\
10.0 \\
1.0 \\
74.0\end{array}$ & $\begin{array}{c}70 \\
7 \\
0 \\
23\end{array}$ & $\begin{array}{c}70.0 \\
7.0 \\
0.0 \\
23.0\end{array}$ & $0.001 * *$ \\
\hline Total & 100 & $100.0 \%$ & 100 & $100.0 \%$ & \\
\hline
\end{tabular}

Mc Nemar test used for pair qualitative variables

(NA) no applicable because response rate $100.0 \%$.

(**) highly statistically significant $p<0.01$

(**) highly statistically significant $p<0.01$ 
Table (5): Foods contain Vitamin $E$ in pre and posttest.

\begin{tabular}{|c|c|c|c|c|c|}
\hline \multirow{2}{*}{ Data related to Foods contain Vitamins $\mathbf{E}$} & \multicolumn{2}{|c|}{ Pre test } & \multicolumn{2}{|c|}{ Post test } & \multirow{2}{*}{-value } \\
\hline & No (100) & $\%$ & No (100) & $\%$ & \\
\hline $\begin{array}{l}\text { 1) Main important to take Vitamin E before } \\
\text { pregnancy: } \\
\text { - Helps in enhances hormone function in women } \\
\text { - Helps increased fertility } \\
\text { - Don't know/ can't remember. }\end{array}$ & $\begin{array}{l}13 \\
16 \\
71\end{array}$ & $\begin{array}{l}13.0 \\
16.0 \\
71.0\end{array}$ & $\begin{array}{c}5 \\
78 \\
17\end{array}$ & $\begin{array}{c}5.0 \\
78.0 \\
17.0\end{array}$ & $0.005 * *$ \\
\hline $\begin{array}{l}\text { 2) Food are naturally good sources of Vitamins E } \\
\text { - Know } \\
\text { - Don't know }\end{array}$ & $\begin{array}{l}37 \\
63\end{array}$ & $\begin{array}{l}36.0 \\
63.0\end{array}$ & $\begin{array}{c}99 \\
1\end{array}$ & $\begin{array}{c}99.0 \\
1.0\end{array}$ & $0.001 * *$ \\
\hline $\begin{array}{l}\text { 3) Adequate dose of Vitamins } \mathrm{E} \text { needed before } \\
\text { pregnancy is: } \\
\text { - } 10 \mathrm{UI} \\
\text { - } 10 \text { milligrams }(\mathrm{mg}) \\
\text { - } 10 \text { micrograms (mcg) } \\
\text { - Don't know. }\end{array}$ & $\begin{array}{l}10 \\
2 \\
5 \\
83\end{array}$ & $\begin{array}{c}10.0 \\
2.0 \\
5.0 \\
83.0\end{array}$ & $\begin{array}{l}70 \\
2 \\
0 \\
28\end{array}$ & $\begin{array}{c}70.0 \\
2.0 \\
0.0 \\
28.0\end{array}$ & $0.002 * *$ \\
\hline Total & 100 & $100.0 \%$ & 100 & $100.0 \%$ & \\
\hline
\end{tabular}

Mc Nemar test used for pair qualitative variables $\quad$ (**) highly statistically significant $p<0.01$

Table (6): Foods contain Caffeine in pre and post test.

\begin{tabular}{|c|c|c|c|c|c|}
\hline \multirow[b]{2}{*}{ Data related to Foods contain Caffeine } & \multicolumn{2}{|c|}{ Pre test } & \multicolumn{2}{|c|}{ Post test } & \multirow{2}{*}{ p-value } \\
\hline & No (100) & $\%$ & No (100) & $\%$ & \\
\hline $\begin{array}{l}\text { 1) Caffeine can lower fertility by approximately } 27 \% \\
\text { - Yes } \\
\text { - No } \\
\text { - Don't know }\end{array}$ & $\begin{array}{c}16 \\
84 \\
0\end{array}$ & $\begin{array}{c}16.0 \\
84.0 \\
0.0\end{array}$ & $\begin{array}{c}99 \\
1 \\
0\end{array}$ & $\begin{array}{c}99.0 \\
1.0 \\
0.0\end{array}$ & $0.001 * *$ \\
\hline $\begin{array}{l}\text { 2) Food are sources of Caffeine } \\
\text { - Know } \\
\text { - Don't know }\end{array}$ & $\begin{array}{l}72 \\
28\end{array}$ & $\begin{array}{c}72.0 \\
0.0\end{array}$ & $\begin{array}{c}100 \\
0\end{array}$ & $\begin{array}{c}100.0 \\
0.0\end{array}$ & NA \\
\hline Total & 100 & $100.0 \%$ & 100 & $100.0 \%$ & \\
\hline
\end{tabular}

Mc Nemar test used for pair qualitative variables

(NA) no applicable because response rate $100.0 \%$

(**) highly statistically significant $p<0.01$

Table (7): Foods contain trans fats in pre and post test.

\begin{tabular}{|c|c|c|c|c|c|}
\hline \multirow{2}{*}{ Data related to foods contain trans fats } & \multicolumn{2}{|c|}{ Pre test } & \multicolumn{2}{|c|}{ Post test } & \multirow{2}{*}{ p-value } \\
\hline & No (100) & $\%$ & No (100) & $\%$ & \\
\hline $\begin{array}{l}\text { 1) Trans fats lower fertility rates } \\
\text { - Yes } \\
\text { - No }\end{array}$ & $\begin{array}{l}19 \\
81\end{array}$ & $\begin{array}{l}19.0 \\
81.0\end{array}$ & $\begin{array}{c}99 \\
1\end{array}$ & $\begin{array}{c}99.0 \\
1.0\end{array}$ & $0.005^{* *}$ \\
\hline $\begin{array}{l}\text { 2) Sources of trans fats food } \\
\text { - Know } \\
\text { - Don't know }\end{array}$ & $\begin{array}{l}54 \\
46\end{array}$ & $\begin{array}{l}54.0 \\
46.0\end{array}$ & $\begin{array}{c}100 \\
0\end{array}$ & $\begin{array}{c}100.0 \\
0.0\end{array}$ & NA \\
\hline Total & 100 & $100.0 \%$ & 100 & $100.0 \%$ & \\
\hline
\end{tabular}

Mc Nemar test used for pair qualitative variables

(NA) no applicable because response rate $100.0 \%$ 


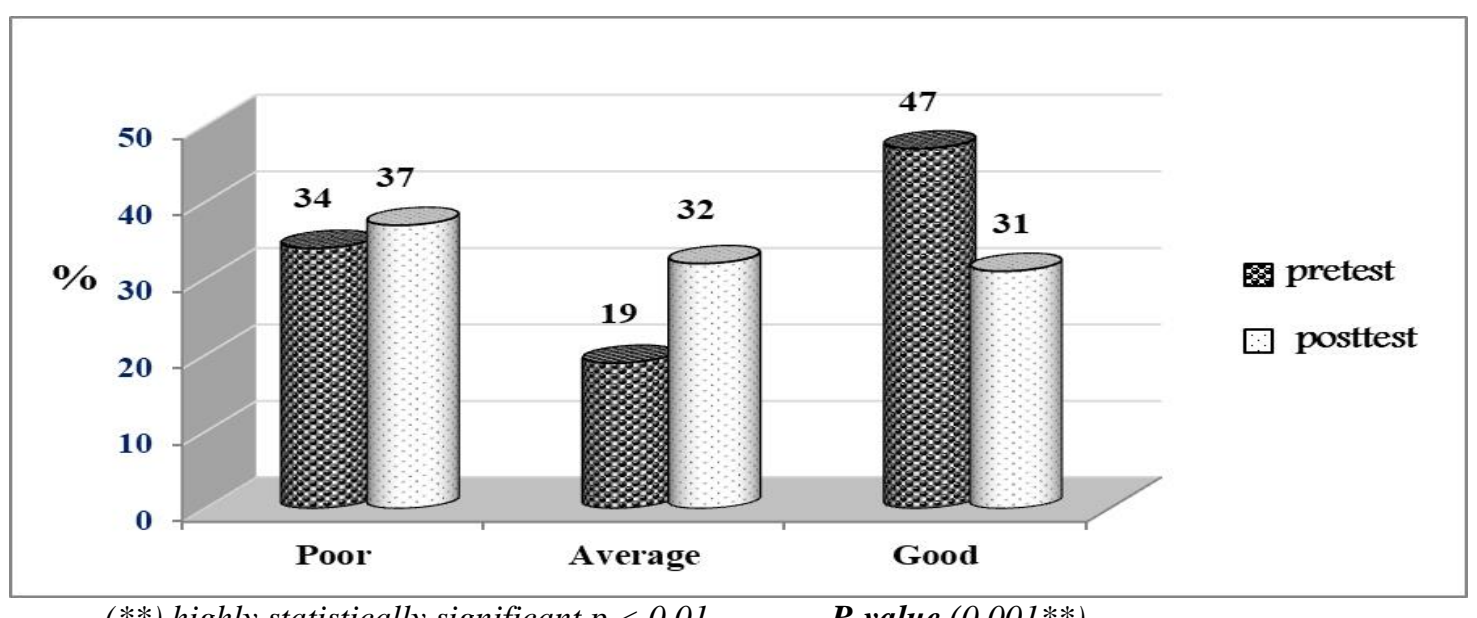

(**) highly statistically significant $p<0.01$

P-value (0.001**).

Figure (1): Total Knowledge in pre and posttest.

Table (8): Relationship between total knowledge in pretest and sociodemographic characteristics of the studied women.

\begin{tabular}{|c|c|c|c|c|c|c|c|c|}
\hline \multirow{3}{*}{$\begin{array}{c}\text { Sociodemographic } \\
\text { characteristics }\end{array}$} & \multicolumn{6}{|c|}{ Total knowledge in pretest } & \multirow{3}{*}{ Total } & \multirow{3}{*}{ P-value } \\
\hline & \multicolumn{2}{|c|}{ Good } & \multicolumn{2}{|c|}{ Average } & \multicolumn{2}{|c|}{ Poor } & & \\
\hline & $\mathbf{N}$ & $\%$ & $\mathbf{N}$ & $\%$ & $\mathbf{N}$ & $\%$ & & \\
\hline $\begin{array}{l}\text { 1) Age/years } \\
\text { - } 18-24 \text { years } \\
\text { - } 25-34 \text { years } \\
\text { - } 35 \text { to } 45 \text { years }\end{array}$ & $\begin{array}{c}6 \\
21 \\
6\end{array}$ & $\begin{array}{c}6.0 \\
21.0 \\
6.0\end{array}$ & $\begin{array}{c}4 \\
13 \\
3\end{array}$ & $\begin{array}{c}4.0 \\
13.0 \\
3.0\end{array}$ & $\begin{array}{c}5 \\
30 \\
12\end{array}$ & $\begin{array}{c}5.0 \\
30.0 \\
12.0\end{array}$ & $\begin{array}{l}15 \\
64 \\
21\end{array}$ & 0.720 \\
\hline $\begin{array}{l}\text { 2) Residence } \\
\text { - Urban } \\
\text { - Rural }\end{array}$ & $\begin{array}{l}19 \\
15\end{array}$ & $\begin{array}{l}19.0 \\
15.0\end{array}$ & $\begin{array}{c}9 \\
10\end{array}$ & $\begin{array}{c}9.0 \\
10.0\end{array}$ & $\begin{array}{l}29 \\
18\end{array}$ & $\begin{array}{l}29.0 \\
18.0\end{array}$ & $\begin{array}{l}57 \\
43\end{array}$ & 0.449 \\
\hline $\begin{array}{l}\text { 3) Educational level } \\
\text { - Illiterate } \\
\text { - Read and write } \\
\text { - Basic education } \\
\text { - Secondary } \\
\text { - University }\end{array}$ & $\begin{array}{l}0 \\
2 \\
4 \\
17 \\
11\end{array}$ & $\begin{array}{l}0.0 \\
2.0 \\
4.0 \\
17.0 \\
11.0\end{array}$ & $\begin{array}{l}1 \\
3 \\
3 \\
7 \\
5\end{array}$ & $\begin{array}{l}2.0 \\
3.0 \\
3.0 \\
7.0 \\
5.0\end{array}$ & $\begin{array}{c}3 \\
7 \\
2 \\
19 \\
16\end{array}$ & $\begin{array}{c}3.0 \\
7.0 \\
2.0 \\
19.0 \\
16.0\end{array}$ & $\begin{array}{c}4 \\
12 \\
9 \\
43 \\
32\end{array}$ & 0.533 \\
\hline $\begin{array}{l}\text { 4) Occupation } \\
\text { - Housewife } \\
\text { - Employed }\end{array}$ & $\begin{array}{l}22 \\
11\end{array}$ & $\begin{array}{l}22.0 \\
11.0\end{array}$ & $\begin{array}{c}12 \\
8\end{array}$ & $\begin{array}{c}12.0 \\
8.0\end{array}$ & $\begin{array}{l}31 \\
16\end{array}$ & $\begin{array}{l}31.0 \\
16.0\end{array}$ & $\begin{array}{l}65 \\
35\end{array}$ & 0.870 \\
\hline $\begin{array}{l}\text { 5) Type of family } \\
\text { - Nuclear } \\
\text { - Extended }\end{array}$ & $\begin{array}{l}15 \\
18\end{array}$ & $\begin{array}{l}15.0 \\
18.0\end{array}$ & $\begin{array}{c}11 \\
9\end{array}$ & $\begin{array}{l}11.0 \\
9.0\end{array}$ & $\begin{array}{l}13 \\
34\end{array}$ & $\begin{array}{l}13.0 \\
34.0\end{array}$ & $\begin{array}{l}39 \\
61\end{array}$ & 0.072 \\
\hline $\begin{array}{l}\text { 6) Age at marriage: } \\
\qquad<20 \text { yrs. } \\
\text { - } 20-25 \text { yrs. } \\
\bullet>25 \text { yrs. }\end{array}$ & $\begin{array}{l}12 \\
13 \\
8\end{array}$ & $\begin{array}{c}12.0 \\
13.0 \\
8.0\end{array}$ & $\begin{array}{l}2 \\
16 \\
2\end{array}$ & $\begin{array}{c}2.0 \\
16.0 \\
2.0\end{array}$ & $\begin{array}{l}15 \\
24 \\
8\end{array}$ & $\begin{array}{l}15.0 \\
24.0 \\
8.0\end{array}$ & $\begin{array}{l}29 \\
53 \\
18\end{array}$ & 0.071 \\
\hline $\begin{array}{l}\text { 7)Duration of marriage: } \\
-<2 \text { yrs. } \\
\text { - } 2-5 \text { yrs. } \\
\text { - }>5 \text { yrs. }\end{array}$ & $\begin{array}{c}1 \\
16 \\
30\end{array}$ & $\begin{array}{c}1.0 \\
16.0 \\
30.0\end{array}$ & $\begin{array}{l}4 \\
8 \\
8\end{array}$ & $\begin{array}{l}4.0 \\
8.0 \\
8.0\end{array}$ & $\begin{array}{c}1 \\
15 \\
17\end{array}$ & $\begin{array}{c}1.0 \\
15.0 \\
17.0\end{array}$ & $\begin{array}{c}6 \\
39 \\
55\end{array}$ & $0.034 *$ \\
\hline Total & 34 & 34.0 & 19 & 19.0 & 47 & 47.0 & & \\
\hline
\end{tabular}

(*) statistically significant p-value $<0.05$ 
Table (9): Relationship between total knowledge in posttest and sociodemographic characteristics of the studied women.

\begin{tabular}{|c|c|c|c|c|c|c|c|c|}
\hline \multirow{3}{*}{$\begin{array}{l}\text { sociodemographic } \\
\text { characteristics }\end{array}$} & \multicolumn{6}{|c|}{ Total knowledge in posttest } & \multirow{3}{*}{ Total } & \multirow{3}{*}{ P-value } \\
\hline & \multicolumn{2}{|c|}{ Good } & \multicolumn{2}{|c|}{ Average } & \multicolumn{2}{|c|}{ Poor } & & \\
\hline & $\mathbf{N}$ & $\%$ & $\mathbf{N}$ & $\%$ & $\mathbf{N}$ & $\%$ & & \\
\hline $\begin{array}{l}\text { 1) } \text { Age/years } \\
\text { - } 18-24 \text { years } \\
\text { - } 25-34 \text { years } \\
\text { - } \quad 35 \text { to } 45 \text { years } \\
\end{array}$ & $\begin{array}{c}9 \\
22 \\
8 \\
\end{array}$ & $\begin{array}{c}9.0 \\
22.0 \\
8.0\end{array}$ & $\begin{array}{c}3 \\
22 \\
7 \\
\end{array}$ & $\begin{array}{c}3.0 \\
22.0 \\
7.0\end{array}$ & $\begin{array}{c}3 \\
20 \\
6 \\
\end{array}$ & $\begin{array}{c}3.0 \\
20.0 \\
6.0\end{array}$ & $\begin{array}{l}15 \\
64 \\
21 \\
\end{array}$ & 0.454 \\
\hline $\begin{array}{ll}\text { 2) } & \text { Residence } \\
\text { - } & \text { Urban } \\
\text { - } & \text { Rural } \\
\end{array}$ & $\begin{array}{l}22 \\
15\end{array}$ & $\begin{array}{l}22.0 \\
15.0\end{array}$ & $\begin{array}{l}14 \\
18 \\
\end{array}$ & $\begin{array}{l}14.0 \\
18.0\end{array}$ & $\begin{array}{l}21 \\
10 \\
\end{array}$ & $\begin{array}{l}21.0 \\
10.0\end{array}$ & $\begin{array}{l}57 \\
43 \\
\end{array}$ & 0.173 \\
\hline $\begin{array}{ll}\text { 3) } & \text { Educational level } \\
\text { - } & \text { Illiterate } \\
\text { - } & \text { Read and write } \\
\text { - } & \text { Basic education } \\
\text { - } & \text { Secondary } \\
\text { - } & \text { University } \\
\end{array}$ & $\begin{array}{c}1 \\
2 \\
5 \\
18 \\
11\end{array}$ & $\begin{array}{l}1.0 \\
2.0 \\
5.0 \\
18.0 \\
11.0\end{array}$ & $\begin{array}{c}1 \\
6 \\
1 \\
14 \\
10\end{array}$ & $\begin{array}{l}1.0 \\
6.0 \\
1.0 \\
14.0 \\
10.0\end{array}$ & $\begin{array}{c}2 \\
4 \\
3 \\
11 \\
11\end{array}$ & $\begin{array}{c}2.0 \\
4.0 \\
3.0 \\
11.0 \\
11.0\end{array}$ & $\begin{array}{c}4 \\
12 \\
9 \\
43 \\
32 \\
\end{array}$ & 0.715 \\
\hline $\begin{array}{l}\text { 4) Occupation } \\
\text { - } \quad \text { Housewife } \\
\text { Employed } \\
\end{array}$ & $\begin{array}{l}24 \\
13 \\
\end{array}$ & $\begin{array}{l}24.0 \\
13.0\end{array}$ & $\begin{array}{l}18 \\
14 \\
\end{array}$ & $\begin{array}{l}18.0 \\
14.0\end{array}$ & $\begin{array}{c}23 \\
8 \\
\end{array}$ & $\begin{array}{c}23.0 \\
8.0 \\
\end{array}$ & $\begin{array}{l}65 \\
35 \\
\end{array}$ & 0.367 \\
\hline $\begin{array}{l}\text { 5) Type of family } \\
\text { - } \quad \text { Nuclear } \\
\text { - } \quad \text { Extended } \\
\end{array}$ & $\begin{array}{l}16 \\
21\end{array}$ & $\begin{array}{l}16 \\
21\end{array}$ & $\begin{array}{l}15 \\
17\end{array}$ & $\begin{array}{l}15 \\
17\end{array}$ & $\begin{array}{c}8 \\
23\end{array}$ & $\begin{array}{c}8 \\
23 \\
\end{array}$ & $\begin{array}{l}39 \\
61 \\
\end{array}$ & 0.234 \\
\hline 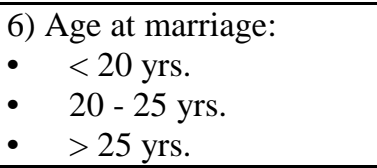 & $\begin{array}{c}12 \\
20 \\
5\end{array}$ & $\begin{array}{c}12.0 \\
20.0 \\
5.0\end{array}$ & $\begin{array}{c}7 \\
17 \\
8 \\
\end{array}$ & $\begin{array}{c}7.0 \\
17.0 \\
8.0\end{array}$ & $\begin{array}{c}10 \\
16 \\
5 \\
\end{array}$ & $\begin{array}{c}10.0 \\
16.0 \\
5.0\end{array}$ & $\begin{array}{l}29 \\
53 \\
18\end{array}$ & 0.676 \\
\hline $\begin{array}{l}\text { 7)Duration of marriage: } \\
\text { - } \quad<2 \text { yrs. } \\
\text { - } \quad 2-5 \text { yrs. } \\
-\quad>5 \text { yrs. } \\
\end{array}$ & $\begin{array}{c}3 \\
15 \\
19 \\
\end{array}$ & $\begin{array}{c}3.0 \\
15.0 \\
19.0\end{array}$ & $\begin{array}{c}2 \\
12 \\
18 \\
\end{array}$ & $\begin{array}{c}2.0 \\
12.0 \\
18.0\end{array}$ & $\begin{array}{c}1 \\
12 \\
18 \\
\end{array}$ & $\begin{array}{c}1.0 \\
12.0 \\
18.0\end{array}$ & $\begin{array}{c}6 \\
39 \\
55\end{array}$ & 0.486 \\
\hline Total & 37 & 37.0 & 32 & 32.0 & 31 & 31.0 & & \\
\hline
\end{tabular}

Table (1): Illustrates that more than half $(64 \%, 57 \%)$ of infertile women are ranged from 23 to 34 years old and live in urban area respectively. About $43 \%$ of them have secondary level of education. Near to double third $(65 \%)$ are housewives and $(61.0 \%)$ of them live in extended family. More than half $(53.0 \%$, $55.0 \%$ ) are married at age 20-25 years and their duration of marriage is more than 5 years respectively.

Table (2): Displays the current infertility \& gynecological data of infertile women, more than half $(54.0 \%)$ of them had a duration of infertility $>5$ years. Regarding causes of infertility the results find that more than one third $(34.0 \%)$ of infertility due to unexplained causes and $(32.0 \%)$ regarding female factors. More than half $(60.0 \%)$ of women free from any gynecological problems but another women complain of health problems, most of these diseases are confined to polycystic ovary syndrome $(24.0 \%)$ and $(7.0 \%)$ had endometriosis.
Table (3): Shows that there are statistical significant differences between pre and posttest regarding importance of foods contains folic acid in increasing fertility, importance of folic acid for fetus, and women need to start folic acid intake P-values are $(0.005,0.02$ and 0.001$)$ for all variables mentioned above respectively.

Regarding food are naturally good sources of folic acid, it's show that P- value is not applicable because $100.0 \%$ of infertile women know about this foods in posttest. There are statistical significant differences between pre and posttests regarding adequate dose of folic acid needed before or through pregnancy, Pvalue is 0.005 .

Table (4): States that there are statistical significant differences between pre and posttest regarding importance of foods contains iron in increasing fertility, important to take iron before pregnancy and adequate dose of iron needed before pregnancy, PValues are $(0.004,0.003,0.001)$ respectively for all variables mentioned above. 
$\mathrm{P}-\mathrm{V}$ alue for food are naturally good sources of iron, is not applicable because response rate $100.0 \%$ in posttest.

Table (5): Reports that there are statistical significant differences between pre and posttest regarding important to take vitamin $\mathrm{E}$ before pregnancy, food naturally good sources of vitamin $\mathrm{E}$ and adequate dose of vitamin $\mathrm{E}$ needed before pregnancy, P-Values are $(0.005,0.001,0.002)$ respectively for all variables mentioned above.

When referring to foods contain caffeine Table (6): Clarifies that there are highly statistical significant differences between pre and posttest regarding effect of caffeine can lower fertility by approximately $27 \%$ $\mathrm{P}-$ Value is 0.001 and foods naturally good sources of Caffeine, P- values are not applicable because $100.0 \%$ of infertile women know about this foods in posttest.

Table (7): Show that there are statistical significant differences between pre and posttest regarding trans fats lower fertility rates $\mathrm{P}$-Value is 0.005 for this variables. And sources of trans fats food, P- values are not applicable because $100.0 \%$ of infertile women know about this foods.

Figure (1): Reveals that there are highly statistical significant differences between pre and posttest regarding total knowledge in pre and posttest, $\mathrm{P}$ Value is 0.001 for this variable.

Table (8): Reveals that there is no statistically significant difference between women's knowledge about food that affect fertility and sociodemographic characteristics of the studied women in pretest except item duration of marriage $\mathrm{P}=0.034$.

Table (9): Reveals that there is no statistically significant difference between total knowledge in posttest and sociodemographic characteristics of the studied women.

\section{Discussion}

The present study aims to assess level of knowledge about nutrition that increases fertility in infertile women, and evaluate effect of educational intervention about nutrition on infertile women's knowledge in preconception period.

The current study reveals that about more than one third of women with good level of knowledge and around one half have poor level of knowledge toward fertility nutrition in pretest. This finding is agree with Mostafa et al., (2013), who applied their study in Saudi Arabia, to assess the knowledge and attitudes of infertile and fertile Saudi participants on infertility, possible risk factors, and social consequences, and reported that more than one half of participants had poor level of knowledge about fertility nutrition.

The present study shows that there is improvement of knowledge of the studied women about fertility nutrition in posttest that about more than one third of women with good level of knowledge. This finding is agree with Mostafa et al., (2013), and reported that more than two third of participants had good level of knowledge about fertility nutrition in posttest. As regard the relationship between the studied women knowledge about nutrition that affect fertility and their sociodemographic characteristics, the present study revealed that about half of those with adequate knowledge had secondary education. These results are consistent with previous research by Riazi et al., (2012), who performed their study in Iran to assess the awareness of pregnant women about folic acid supplementation, who denoted that there was a significant relationship between knowledge and education. This informs that high education can affect women's ability to increase their knowledge and information.

Insufficient folate intake was reported to cause the luteal progesterone levels to decrease and the risk of anovulation to increase Kelton \& Karma, (2015). The present study reveals that there is a highly statistical significant difference between knowledge of participants in the pre and posttest regarding food that contains folic acid and its importance to increase fertility. This finding is similar to the result of Jolanda (2008), who performed their study in Netherlands to identify the effect of folic acid treatment on the microenvironment of the maturing oocyte in humans, and reported that there was a highly statistically significant difference between influence of folic acid supplementation on the follicular fluid concentrations of folate and total homocysteine and their relationship to the diameter of the follicle that thereby influence the microenvironment of the maturing oocyte, $\quad(p \leq$ 0.001).In the end it is obvious that folic acid supplementation is very important to enhance women's fertility.

According to (Kelton \& Karma, 2015) who reported that intake of nonheme iron was found to be related to lower risk of infertility due to anovulation. The current study reveals that knowledge about foods contains iron are important to increase fertility, it is important to take iron before pregnancy and adequate dose of iron needed before pregnancy, There are statistical significant differences between pre and posttest, P-Values are $(0.004,0.003,0.001)$ respectively. This finding is agreed with Rakesh et al., (2013), who applied their study to demonstrate the potential effects of multiple lifestyles on reproductive health for both men and women in USA, and found that an increased intake of iron and multivitamins had lower rates of infertility due to ovulation disorders $(\mathrm{p}<0.001)$. 
Vitamin E was initially denoted as an "anti-sterility factor X" that was necessary for reproduction (Siti et al., 2018). The present study reveals that there is a highly statistical significant difference between knowledge of participants in the pre and posttest regarding important to take vitamin $\mathrm{E}$ before pregnancy. This finding in the same line with Elizabeth et al., (2014), who implemented their study to determine whether increased antioxidant intake in women is associated with shorter time to pregnancy among a cohort of couples being treated for unexplained infertility in Pennsylvania, and illustrated that increased intake of vitamin $\mathrm{E}$ from dietary supplements and total vitamin $\mathrm{E}$ intake were associated with shorter time to pregnancy in women $(\mathrm{p}<0.001)$.

As regard to nutrients that affect negatively on women fertility and must be avoided as caffeine and trans fats, Audrey \& Jorge, (2018) stated that caffeine may affect female reproduction by targeting ovulation and corpus luteal function through alterations to hormone levels and has been associated with higher early follicular E2 levels in females (Peerkhan, 2014). The present study clarifies that there are highly statistical significant differences between pre and posttest regarding knowledge related caffeine it can lower fertility by approximately $27 \%$, and foods are naturally good sources of Caffeine, PValue is 0.001. This finding is agreed with Rakesh et al, (2013), who reported that caffeine has negative effects on female fertility. Caffeine has been associated with an increase in the time to pregnancy of over 9.5 months, particularly if the amount is over $500 \mathrm{mg}$ per day (OR 1.45; 95\% CI1.03-2.04).

Moreover, finding of this study were disagree with Chavarro et al., (2009), who performed their study in USA, to identify relationship between ovulatory disorder infertility and caffeinated and alcoholic beverage intake. They found that neither alcohol nor caffeine intake appeared to impair ovulation to the point of decreasing fertility.

Artificial fats, such as trans fats, can disrupt fertility by increasing insulin insensitivity and inflammation in the body, interfering with ovulation, conception, and early embryonic development (Mary, 2013) The present study shows that there are statistical significant differences between pre and posttest regarding knowledge of participants related to trans fats lower fertility rates P-Value is 0.005 . This finding is agree with Rakesh et al., (2013), who demonstrate that consuming trans fats instead of carbohydrates correlated with a $73 \%$ increase in risk of ovulatory disorder.

The current study shows that more than half of participants are married at age 20-25 years and their duration of marriage is more than 5 years. This was in conformity with Leah et al., (2014), who applied their study to assess perceptions among infertile couples of lifestyle behaviors and in vitro fertilization (IVF) success in USA, who reported that more than half $(50.4 \%)$ of participant women number of months trying to get pregnant 5 years.

As regard to sociodemographic characteristics of studied women, the present study shows that more than half of infertile women their age is ranged from 23 to 34 years old, more than one third of them have secondary level of education. These finding agree with Tiina et al., (2017), who implement their study in Sweden, to investigate folic acid supplement use and folate status among women under treatment for infertility (hereafter infertile) and fertile women also in regard to socioeconomic and lifestyle factors, the age of women were 22- 34 years $(55.8 \%)$ and (43.8) have secondary level of education .

In the current study near to double third are housewives. These findings agree with Guliz \& Yilda, (2015), who performed their study in Turkey, to identify relation between anxiety and healthy lifestyle on IVF outcomes, who found that near to two thirds were housewives. These findings are not consistent with Riazi et al., (2012), he found that $32.9 \%$ of the studied women their age ranged from 20-24 years old, while the majority of individuals $(42.9 \%)$ had elementary education, and in the line with the same investigator regarding occupation who stated that most of respondents (95.7\%) were housewives.

Regarding current infertility \& gynecological data of infertile women, present study reveals that more than half of them had a duration of infertility $>5$ years. This was in conformity with Guliz\& Yilda, (2015) who reported that more than one third $(37.3 \%)$ of participant women had duration of 6-10 years of infertility.

According to causes of infertility the results of actual find that more than one third of infertility due to unexplained causes and nearly one third regarding female factors. This finding on the same line with Leah et al., (2014) he found that causes of infertility due to unexplained causes were $(37.0 \%)$ and $(35 \%)$ regarding female factors.

\section{Conclusion}

Educational program about fertility nutrition is very important to enhance infertile women's knowledge regarding nutrition that increase and decrease fertility. After implementing this study, its finding can answer the research questions as this study cannot assume that women are knowledgeable about fertility nutrition and its health benefits. 


\section{Recommendation}

The study recommended that implement special measures as: A) develop health education program for the population based on its cultural and sociodemographic characteristics. This program could be simple as providing information on the benefits of fertility nutrition sources using all available mass media as (posters, magazine, and brochures in Maternity and Child Health (MCH) Centers.

Acknowledgement

The authors gratefully acknowledge the contribution of the infertile women in the study.

\section{References}

1. Abdallah Eldib, Osama A., Tashani (2018): Infertility in the Middle East and North Africa Region: A systematic review with metaAnalysis of prevalence surveys, Volume: 2 | Issue : 2 | Page : 37-44.

2. American Pregnancy Association (2018): Preconception Nutrition for Increasing Your Fertility. Available at: http://americanpregnancy.org/gettingpregnant/preconception-nutrition.

3. American Pregnancy Association (2019): How Do You Get Pregnant Naturally, available at: https://americanpregnancy.org/planning/getpregnant-naturally

4. American Society for Reproductive Medicine (2019): Defining Infertility, available at: https://www.reproductivefacts.org.

5. Audrey J. Gakins \& Jorge Chavarro (2018): the relationship between diet and human fertility, Volume 218, Issue 4, Pages 379-389.

6. Centers for Disease Control and Prevention (2013): Infertility FAQs. Available at: https://www.cdc.gov /reproductivehealth/ infertility/index.htm.

7. Chavarro J., Rich-Edwards J., Rosner B., Willett W., (2009): Caffeinated and alcoholic beverage intake in relation to ovulatory disorderinfertility. Epidemiology 20:374-81.

10.1097/EDE.0b013e31819d68cc [PMC free article] [PubMed] [CrossRef] [Google Scholar]

8. Elizabeth H., Ruder, Terryl J., Hartman, Richard H., Reindollar, \& Marlene B., Goldman, (2014): Female dietary antioxidant intake and time to pregnancy among couples treated for unexplained infertility, 101(3): 759766.

9. Erica Silvestris, Domenica Lover, \& Raffaele Palmirotta (2019): Nutrition and Female Fertility: An Interdependent Correlation, 10:346. doi: 10.3389/fendo.2019.00346, available at: https://www.frontiersin.org/articles.
10. Gaskins A., \& Chavarro J., (2018): Diet and fertility: a review. Am J Obstet Gynecol. 218:379-89. 10.1016/j.ajog.2017.08.010 [PMC free article] [PubMed] [CrossRef] [Google Scholar]

11. Güliz Onat \& Yılda Arzu Aba (2015): the effects of a healthy lifestyle and of anxiety levels on IVF outcomes, 19(4):92-101.

12. Hethir R., (2019): Harvard shows $80 \%$ Reduction in Infertility from Natural Lifestyle Changes available at: http://www.hsph.harvard.edu/news/press.

13. Hillstrom K., (2018): nutrition across life stages, nutrition recommended during preconception, $1^{\text {st }}$ edition, Elsevier comp, page 44.

14.Homan G., \&Norman R., (2018): Couples perception regarding how lifestyle might affect fertility: results of a pilot study. Aust $\mathrm{J}$ Adv Nurs 26:77-86.

15.Jolanda C., Boxmeer (2008): The homocysteine pathway, female fertility and IVF outcomes Fertil Steril. 89(6):1766-70.

16. Kelton Tremellen \& Karma Pearce (2015): Nutrition, Fertility, and Human Reproductive Function, $1^{\text {st }}$ edition, chapter 1 , Nutrition and Ovulatory Function, page 5-6, 12.

17. Khosrorad T., Dolation M., Shahsavari S., \& Bakhtiari M., (2015): Comparison of lifestyle in fertile and infertile couples in Kermanshah during 2013. Iran J Reprod Med 13:549-556.

18. Leah K., Hawkins, Brooke V. Rossi, Katharine F., Correia, Shane T., Lipskind, Mark D., Hornstein \& Stacey A., Missmer (2014): Perceptions among infertile couples of lifestyle behaviors and (IVF) success, J Assist Reprod Genet 31, 255-260 doi:10.1007/s10815014-0176-5.

19. Mary D., (2013): Optimizing female fertility through diet. Available at: https://www.eggnutritioncenter.org.

20. Mostafa A., Abolfotouh, Abdullah A., Alabdrabalnabi, Rehab B., Albacker, Umar A., Al-Jughaiman \& Samar N., Hassan (2013): Knowledge, attitude, and practices of infertility among Saudi couples, 563-573, International Journal of General Medicine 2013:6.

21.Peerkhan Nazni (2014): Association of western diet \& lifestyle with decreased fertility, pp 7881. Indian J Med Res 140 (Supplement).

22.Philip B., Imler \& David Wilbanks (2018): the essential guide to getting pregnant, essential nutrients for women chapter $4,1^{\text {st }}$ edition, Amazon comp, page 25. 
23. Rakesh Sharma, Kelly R., Biedenharn, Jennifer M., Fedor \& Ashok Agarwal (2013): Lifestyle factors and reproductive health: taking control of your fertility, 11:66, doi:10.1186/1477-7827-11-66

24. Riazi, H., Bashirian, S., \& Amini, L., (2012): Awareness of Pregnant Women about Folic Acid Supplementation in Iran Faculty of Nursing \& Midwifery, Shahid Beheshti University of Medical Sciences, Tehran, Iran , Journal of Family and Reproductive Health 6( 4).

25. Rizk B., Olsen M., (2016): Challenging office cases in obstetrics and gynecology. Johnson City: Jaypee Brothers Medical Publishers Ltd; pp. 65-65, available at: https://www.hhs.gov/opa/reproductivehealth/fact-sheets/female-infertility/index.html

26. Siti Syairah Mohd Mutalip, Sharaniza AbRahim, \& Mohd Hamim Rajikin(2018): Vitamin $\mathrm{E}$ as an Antioxidant in Female Reproductive Health, 7(2): 22.

27. Tiina Murtoa, Agneta Yngveb, Agneta Skoog Svanberga, Signe Altmäec, Andres Salumetsc, D., Kjell Wånggreng \& Anneli Stavreus-Eversa (2017): Compliance to the recommended use of folic acid supplements for women in Sweden is higher among those under treatment for infertility than among fertile controls and is also related to socioeconomic status and lifestyle, VOL. 61, 1334483 https://doi.org/10.1080/16546628.2017.1334483

28. U.S. Department of Agriculture, Agricultural Research Service. FoodData Central (2019): vitamins. Available at: https://www.nal.usda.gov/fnic

29.U.S. Department of Health and Human Services (2019): Infertility, available at: https://www.womenshealth.gov/a-ztopics/infertility.

30. Virpi Kemppainen, Kerttu Tossavainen, \& Hannele Turunen (2013): Nurses' roles in health promotion practice: an integrative review, Volume 28, Issue 4, and Pages 490-501.

31. Yoo S., \& Sobotka T., (2018): Ultra-low fertility in South Korea: The role of the tempo effect, Vol. 38.22, pp. 549-576. 